\title{
How Do Energies Complexify?
}

H. Bíla

Some particular properties of the parametric dependence of eigenvalues with emphasis on their complexification are discussed. The non-diagonalisability of $\mathcal{P}$ T-symmetric matrix Hamiltonians in exceptional points is compared with level-crossing prohibition of Hermitian systems. For non-matrix Hamiltonians, the different way of complexification between Klein-Gordon and Dirac Hamiltonians is demonstrated.

Keywords: $\mathcal{P}$ T-symmetry, exceptional points, Dirac equation

\section{Introduction}

In physics there are usually one or more free parameters which specify some properties of the system. Aside from trivial parametric dependences that can be determined directly, e.g., by a change of units, variation in the values of some parameters can qualitatively affect the system's behaviour. In quantum physics, where almost all physical properties are related to spectral properties of a chosen set of operators (in particular the Hamiltonian), qualitative changes of spectra due to varying parameters of the Hamiltonian are of prominent importance.

$\mathcal{P}$-symmetry, which was introduced as a concept generalising the usually demanded self-adjointness of the Hamiltonian, does not per se provide the reality of its spectrum and consequently of the energies. Even if the spectrum is real it need not to be so for all values of the considered parameters. Therefore determining the regions in the parametric space where the spectrum's reality holds is a key step in finding the limits of the physical significance of any $\mathcal{P} \mathcal{T}$-symmetric theory ${ }^{(1)}$.

Most attention has been concentrated on discrete spectra, for the following reasons. First, it is usually more convenient (at least for mathematically less careful and less rigorous physicists) to deal with isolated eigenvalues than with the continuous part of the spectrum, where all manipulations become in a way more mathematically intricate. Second, there are many systems whose spectrum is fully discrete and others where the essential spectrum is insensitive to parametric change. The points of eigenvalues' complexification are thus the investigated limits of physical relevance. After examining many of the "classical" examples of $\mathcal{P} \mathcal{T}$-symmetric systems an apparently regular pattern was observed - a square-root singularity structure and a Jordan-block degeneracy.

Though this is beyond the scope of this article, it can be useful for the reader to consider one more application of studying complexification and its properties. Complex (albeit not necessarily $\mathcal{P} \mathcal{T}$-symmetric) Hamiltonians do also arise with extending parameters of the Hermitian systems into the complex domain. The structure of the spectrum and especially the distribution of the exceptional points (which are, in this case up to slight generalisation, the points of the spectrum's complexification) then influences the phase transitions and the chaotic behaviour of many systems. See e.g. [2] or [3] (and refs. therein).
Outside of quantum mechanics, $\mathcal{P}$-symmetric Hamiltonians are of use in the framework of magnetohydrodynamics. Here again the exceptional points and level crossings (we will discuss their close relatedness later) have a direct physical significance. For the connection between magnetohydrodynamics and $\mathcal{P} \mathcal{T}$-symmetry, see $[4,5]$. The concrete nature of the complexification is important, e.g., if one has to construct a perturbation theory around these singularities [6].

The purpose of this paper is to summarise some well-known facts about complexification of energies and also to discuss some of the other (than usual) possible scenarios of complexification, especially for Dirac operators. In the first section we will make clear the terminology and compare particular properties of self-adjoint and $\mathcal{P} \mathcal{T}$-symmetric parametrically dependent Hamiltonians. In the second section, we will present the situation in relativistic models.

\section{The basics of complexification}

In the following, we will investigate the spectra of operators that depend on a single parameter. These operators will be called Hamiltonians, as is usual in the $\mathcal{P} \mathcal{T}$-symmetric context, even if the matrices used for illustration in this section are rather toy models than realistic generators of time evolution. However, the physical background of what is discussed in the second section will be rather obvious.

The investigated parameter will be denoted by $c$, the Hamiltonian $\mathrm{H}(c)$ and its eigenvalues $E$, unless stated otherwise. The point in the parametric space where complexification occurs will be called the exceptional point ${ }^{(2)}$ (EP) and denoted by $c_{\text {ep }}$; complexification means that some chosen subset (usually consisting of two eigenvalues) of the spectrum $\sigma(\mathrm{H}(c))$ is real for $c<c_{\mathrm{ep}}$ and imaginary for $c>c_{\mathrm{ep}}$ or vice versa.

To avoid unnecessary complications we will examine the most common situation when the Hamiltonian is of the form

$$
\mathrm{H}(c)=\mathrm{H}_{0}+c \mathrm{~V}
$$

Note that in spite of being quite restrictive within all imaginable parametric dependences, form (1) is useful in a broad class of physical applications. Obviously, one can get other than linear parametric dependences by means of reparametrisation. 
All discussed Hamiltonians will be $\mathcal{P}$ T-symmetric. This means that there exists an anti-linear operator, conventionally written as a product of time reversal $\mathcal{T}$ and parity reflection $\mathcal{P},{ }^{(3)}$ which commutes with the Hamiltonian:

$$
\mathcal{P} \mathcal{T} \mathrm{H}(c)=\mathrm{H}(c) \mathcal{P} \mathcal{T}
$$

It is well known that condition (2) itself provides that the discrete spectrum consists of real eigenvalues and complex-conjugated pairs. Thanks to the simple parametric dependence of $\mathrm{H}$ it is reasonable to estimate that the eigenvalues are continuous functions of $c{ }^{(4)}$ From these two facts one can infer that at the exceptional point at least two eigenvalues must merge. Hence it could be useful to generalise the notion of EPs to those $c$ where the spectrum is degenerated even if it is real (and non-degenerated) in the neighbourhood of the point.

\subsection{Matrices and avoided crossings}

The simplest case of a $\mathcal{P} \mathcal{T}$-symmetric system is a parametrically dependent (finite-dimensional) matrix. Examples of these have been used many times to demonstrate various properties of $\mathcal{P} \mathcal{T}$-symmetric systems. For some infinite-dimensional systems, non-self-adjointness is essential only in the subspaces spanned on the lowest-energy eigenvectors [7, 8] and matrix representations of the Hamiltonian on this subspace can be considered as a good approximation to the complete problem.

Because for matrices of form (1) the eigenvalues are always continuous functions of $c$ and their total number is fixed, complexification can clearly happen only in two ways. In the first case, at the exceptional point there is an $n$-dimensional degenerate subspace with $n$ independent eigenvectors. In the second case, there are only $n-k$ independent eigenvectors with $k>0$ and there is thus a Jordan-block structure.

The important thing now is that the former case, let us call it diagonalisable EP, is much rarer than the latter (non-diagonalisable). To see this, let us first examine the simplest $2 \times 2$ matrix case. A two-dimensional diagonalisable matrix with a degenerated spectrum is a multiple of the unit matrix and so if one assumes diagonalisable EP at $c_{\text {ep }}$, then $\mathrm{H}\left(c_{\text {ep }}\right)=k \mid$ and thus

$$
\mathrm{H}_{0}=k \mathrm{l}+\left(c-c_{\mathrm{ep}}\right) \mathrm{V} \text {. }
$$

The consequence is that $\mathrm{H}_{0}$ and $\mathrm{V}$ commute. This is something that is not satisfied in almost all physical situations. However it must be emphasised that although the commutativity of $\mathrm{H}_{0}$ and $\mathrm{V}$ is a sufficient condition for the existence of diagonalisable EPs in any dimension of Hilbert space, it is a necessary condition for that only in the case of two dimensional systems. The stated argument cannot be easily generalised to more dimensions, and it needs a different approach to see the reasons why diagonalisable EPs are "prohibited".

Hermitian matrices are always diagonalisable and thus the only EP that can occur in a Hermitian parametrically dependent system is a diagonalisable one, which is usually referred to as a level crossing. That level crossings are in some way avoided for Hermitian systems is well known, but because the mechanism of this is exactly the same as the mechanism protecting against diagonalisable complexification in the $\mathcal{P} \mathcal{T}$-symmetric case, let us formulate the statement more precisely. For the purposes of this article, the following formulation of the "no-crossing" theorem will be sufficient:

Let $\mathcal{V}$ be a set of all $N$-dimensional Hermitian matrices and $S: \mathcal{V} \rightarrow \mathbb{R}^{N^{2}}$ be a mapping that maps any $\mathrm{V} \in \mathcal{V}$ with entries $v_{i j}$ to the vector

$S(\mathrm{~V})=\left(v_{11}, \mathfrak{R} v_{12}, \mathfrak{I} v_{12}, \ldots, \mathfrak{J} v_{1 N}, v_{22}, \mathfrak{R} v_{23}, \ldots, v_{N N}\right)$.

Let $\mathrm{H}_{0}$ then be a fixed Hermitian operator and $\mathcal{V}_{\mathrm{C}}$ be a subset of $\mathcal{V}$ such that for all $\mathrm{V} \in \mathcal{V}_{\mathrm{C}}$ the operator $\mathrm{H}_{0}+c \mathrm{~V}$ has a level crossing for at least one $c \in \mathbb{R}$. Then $S\left(\mathcal{V}_{\mathrm{C}}\right)$ has zero measure in $\mathbb{R}^{N^{2}}$.(5)

The message of the theorem is that parametrically dependent matrices of the form (1) which have a level crossing are extremely rare in the sense that they constitute a zero-measure subset of all possible matrices of the considered type. A sketch of the proof can be outlined as follows: The characteristic polynomial

$$
\chi(E)=\operatorname{det}\left(\mathrm{H}_{0}+c \mathrm{~V}-E \mathrm{I}\right)
$$

of a Hermitian matrix is real for all real $c$ and $E$. It must have a multiple root in an exceptional point, which is otherwise stated as

$$
\begin{aligned}
& \chi\left(c_{\mathrm{ep}}\right)=0, \\
& \left.\frac{\partial \chi}{\partial E}\right|_{c_{\mathrm{ep}}}=0 .
\end{aligned}
$$

The dependence of $\chi$ on $c$ is polynomial and thus smooth and therefore also

$$
\left.\frac{\partial \chi}{\partial c}\right|_{c_{\mathrm{ep}}}=0 ;
$$

otherwise $\chi$ would have complex roots at $c_{\mathrm{ep}}+\varepsilon$ for some non-zero small $\varepsilon$.

Now, having a given $\mathrm{H}_{0}$, let us choose any $c_{\text {ep }}$ and $E_{\text {ep }}$ and find any matrix $\mathcal{V}$ such that $\mathrm{H}_{0}+c \mathrm{~V}$ has an EP at $c_{0}$ with the energy $E_{\text {ep }}{ }^{(6)}$ One may ask what happens with the eigenvalues if the matrix elements of $\mathrm{V}$ (or better the components $x_{i}$ of the vector $\left.x \equiv S(\mathrm{~V})\right)$ and the parameter $c$ are slightly varied around the selected point. In the $\left(N^{2}+2\right)$-dimensional space of variables $x_{i}, c$ and $E$ the eigenvalues are located on the subset where $\chi(x, c, E)$, therefore one needs to solve the differential equation

$$
0=\mathrm{d} \chi=\left(\nabla_{x} \chi\right) \mathrm{d} x+\frac{\partial \chi}{\partial E} \mathrm{~d} E+\frac{\partial \chi}{\partial c} \mathrm{~d} c .
$$

From (6) and (7) it follows that if one starts at the EP the last two terms in (9) vanish and one gets

$$
0=\left(\nabla_{x} \chi\right) \mathrm{d} x
$$

This is an equation which defines an $\left(N^{2}+2\right)$ dimensional manifold in $\mathbb{R}^{N^{2}}$. It is obvious now that the $N^{2}$-dimensional vectors $x$ for which $S^{-1}(\boldsymbol{x}) \in \mathcal{V}_{\mathrm{C}}$ are restricted to lie on such manifolds clearly of zero measure since they have one dimension less than the space of all Hermitian $N \times N$ matrices. ${ }^{(7)}$ 
What are the differences if one takes the $\mathcal{P} \mathcal{T}$-symmetric case instead of Hermitian one? The number of free real parameters determining a $\mathcal{P} \mathcal{T}$-symmetric matrix is the same as for a Hermitian matrix and its characteristic polynomial is also real. The crucial difference is that since there is no guaranteed reality of the spectrum, condition (8) does not hold. On the other hand since we know [9] that a diagonalisable $\mathcal{P T}$-symmetric matrix with real eigenvalues can be transformed to a Hermitian one by a similarity transformation, and because similar matrices have the same characteristic polynomials, condition (8) is still valid for diagonalisable EPs of $\mathcal{P} \mathcal{T}$-symmetric matrices. However the argument does not apply for non-diagonalisable EPs, where an analogous similarity transformation does not exist. In this case, (9) can be solved by a function $c(\boldsymbol{x})$ that determines the position of the selected EP under an arbitrary change of parameters $x_{i}$.

\subsection{Square-root dependence}

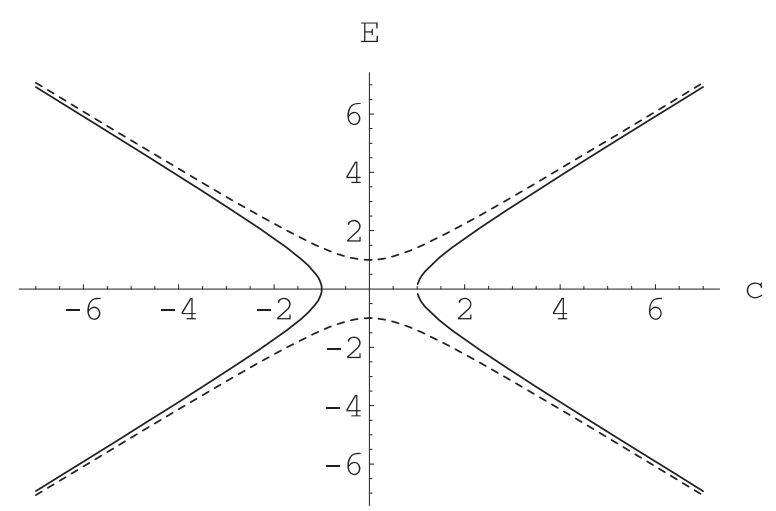

Fig. 1: The eigenvalues of matrix (11) with $c_{1}=c_{2}=c$ and $d=e^{i q}=1$ (solid lines). The dashed lines represent an analogous Hermitian matrix where both off-diagonal elements are +1 . The graph illustrates the typical behaviour of energy levels of both Hermitian systems (avoided crossing at $c=0$ ) and $\mathcal{P} \mathcal{T}$-symmetric systems (complexification at exceptional points $c= \pm 1$ ).

To illustrate the behaviour of eigenvalues on a concrete example, let us consider the simple two-dimensional matrices

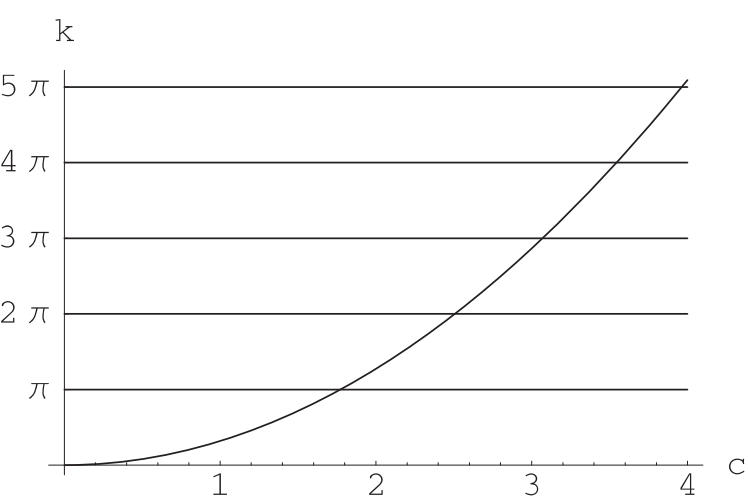

$$
\mathrm{H}=\left(\begin{array}{cc}
c_{1} & d e^{i q} \\
-d e^{-i q} & -c_{2}
\end{array}\right)
$$

with $c_{i}, d, q \in \mathbb{R} .^{(8)}$ The eigenvalues are given by

$$
2 E=c_{1}-c_{2} \pm \sqrt{\left(c_{1}+c_{2}\right)^{2}-4 d^{2}} .
$$

The square root in (12) is a typical example of parametric dependence near the non-diagonalisable EP. Since (11) is a very simple (i.e. two-level) system the dependence is exactly the square root; for larger matrices of type (1) the square-root function is modified by some non-singular $c$-dependent factor, but still

$$
\lim _{c \rightarrow c_{\mathrm{ep}}} \frac{\mathrm{d} E}{\mathrm{~d} c}= \pm \infty .
$$

The essential fact is that in complex neighbourhood of the exceptional point the eigenvalues form a structure with two Riemann sheets typical for the square root - this is universally true for matrices in all EPs where two levels cross. The scheme also holds for a large class of Schrödinger operators with $\mathcal{P} \mathcal{T}$-symmetric potentials. A square-root singularity was attested for example in the founding problem of $\mathcal{P} \mathcal{T}$-symmetry - the Hamiltonians

$$
-\frac{\mathrm{d}^{2}}{\mathrm{~d} x^{2}}-(i x)^{2+c}
$$

of Bender, Boettcher and Meisinger [10]. Another example is the one-dimensional Laplacian at the interval $(0, d)$ discussed in [11] with $\mathcal{P} \mathcal{T}$-symmetric boundary conditions ${ }^{(9)}$

$\psi^{\prime}(0)=-(b+i c) \psi(0), \quad \psi^{\prime}(d)=(b-i c) \psi(d)$

The system defined in such a way has several interesting properties: The total number of complex eigenvalues does not exceed one pair. The exceptional point usually has a Jordan-block degeneracy and the square-root-like behaviour of the energy, but the situation can be made slightly more complicated for example by keeping $\sqrt{b^{2}+c^{2}}=n+1 / 2$ fixed and varying $c$, which leads to unusual triple (still non-diagonalisable) degeneracy in the EP.

An even more extraordinary possibility is to put $b=0$ and observe the dependence on $c$. In this setting the spectrum is real for all $c$ and only one energy level is not constant; the exceptional points occur at $k=n \pi$, where $n \in \mathbb{N}$ and $k=\sqrt{E}$.

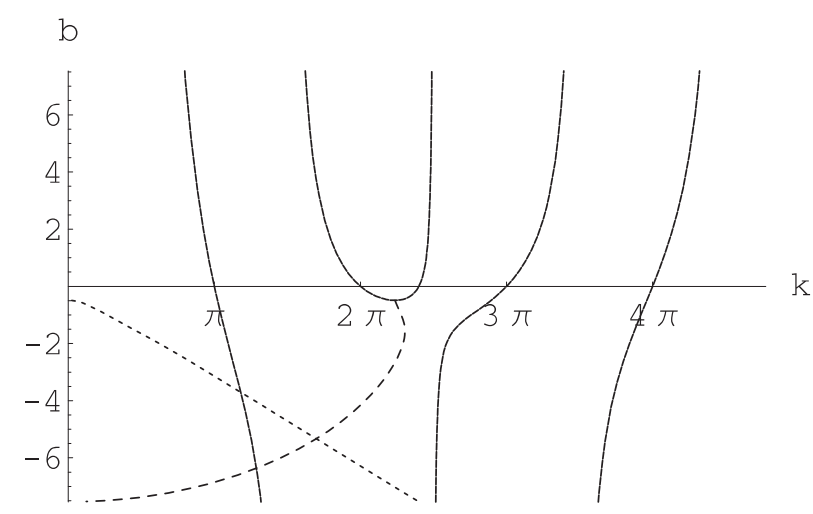

Fig. 2: The energy dependence of the Laplacian with boundary conditions (15). The left graph shows the level crossings for $b=0$. The situation on the right represents varying $b$ for fixed value $\sqrt{b^{2}+c^{2}}=2.4$. Solid lines represent real eigenvalues while dashed and dotted lines are the real and imaginary parts of one of the complex eigenvalues respectively (the second one is conjugated). The values of the wave vector $k=\sqrt{E}$ are shown. 
Because actually no complexification occurs also (13) does not hold, but a Jordan-block structure is still maintained.

As a summary to the first section it is worth noting that the merging of two (or ocassionally more) eigenstates and eigenvalues in the point of complexification, while being obligatory for matrices, is prevalent but not universal for Schrödinger operators. Complications come into play when the potential involves a singularity, typically a divergent term proportional to $1 / x$. In such situations the eigenfunction corresponding to one of the real eigenvalues expected to merge at the EP ceases to be square integrable and thus in this point one sees a splitting of one real energy into two complex energies (see for example [12]). We will also discuss this later with the radial Dirac equation.

\section{Relativistic systems}

The equations of relativistic quantum mechanics provide a natural resource of systems with complexification phenomena. In contrast to non-relativistic $\mathcal{P} \mathcal{T}$-symmetry, here one does not need to add an "artificial" complex term and complexification is encountered within undoubtedly physically relevant problems. The point of complexification is usually regarded as the furthermost boundary of quantum-mechanically describable configuration. In the following subsections we will discuss in more detail three different relativistic models.

\subsection{Dirac square well}

One of the simplest interactions that can be imagined is represented by a finite square well potential. The first discussed relativistic system will be the spin $1 / 2$ particle in such potential. The one-dimensional Dirac Hamiltonian in one suitable representation reads

$$
\mathrm{H}=\left(\begin{array}{cc}
m+c \chi_{(0, d)}(x) & -i \partial_{x} \\
-i \partial_{x} & -m+c \chi_{(0, d)}(x)
\end{array}\right),
$$

where $c$ and $d$ are the well's depth and width, respectively, and is the mass of the particle, throughout the following deliberately put to be equal to one. The domain of the Hamiltonian (16) is

$$
\operatorname{DomH}=\left\{\psi \in L_{2}(\mathbb{R}) \oplus L_{2}(\mathbb{R}) \mid \psi^{\prime} \in L_{2}(\mathbb{R}) \oplus L_{2}(\mathbb{R})\right\} .
$$

The Hamiltonian has as usual the Dirac Hamiltonian continuous spectrum $\sigma_{c}=(-\infty,-1) \cap(1, \infty)$ which does not depend on $c, d$. Our interest is concentrated on the bound states. The search for stationary states consists of solving the problem on the intervals $I_{\mathrm{I}}=(-\infty, 0), I_{\mathrm{II}}=(0, d)$ and $I_{\mathrm{III}}=(d, \infty)$. The normalisable solutions for energy $E$ are

$$
\begin{aligned}
& \psi_{\mathrm{I}}=K_{\mathrm{I}} \mathrm{e}^{\Omega x}, \\
& \psi_{\mathrm{II}}=K_{\mathrm{II}}^{+} \mathrm{e}^{\omega x}+K_{\mathrm{II}}^{-} \mathrm{e}^{-\omega x}, \\
& \psi_{\text {III }}=K_{\text {III }} \mathrm{e}^{-\Omega x} .
\end{aligned}
$$

where $K$ refers to still unspecified complex constants and

$$
\begin{aligned}
& \rho_{ \pm}=\sqrt{1 \pm(c+E)}, \\
& P_{ \pm}=\sqrt{1 \pm E}, \\
& \omega=\rho_{+} \rho_{-}, \\
& \Omega=P_{+} P_{-},
\end{aligned}
$$

the square roots are taken positive or positive imaginary (that is purely imaginary with positive imaginary parts). The matching conditions in 0 and $d$ involve then only the wave-function, not the derivative, as $\mathrm{H}$ contains only the first derivative term. After eliminating all $K \mathrm{~s}$ we get the equation

$$
(1+\xi) \mathrm{e}^{\omega d}=1-\xi
$$

with $\xi=\frac{P_{-} \rho_{+}}{P_{+} \rho_{-}}$. From the definitions above it can be seen that $\xi$ is real positive or imaginary positive, but no real positive $\xi$ can fulfill equation (20). It follows that $E$ lies in the interval $(-1,1)$ and thus in the gap of the continuous spectrum, as might be expected. Now it would be useful to take a logarithm of both sides of (20) to get a real equation

$\frac{\pi}{2}-\arctan \sqrt{\frac{(1+E)(c+E-1)}{(1-E)(c+E+1)}}=\frac{d}{2} \sqrt{(c+E)^{2}-1} \bmod \pi$.

By investigating (21) we arrive at the following observations about the dependence of the energies on parameters $c$ and $d$ :

1. Due to the symmetry of the system the change $c \rightarrow-c$ yields $E \rightarrow-E$ and thus one can restrict one's attention to $c>0$ without loss of generality.

2. Each energy decreases as $c$ or $d$ increases.

3 . For the $i$-th bound state and fixed $d$ there are critical values $c_{i}^{\min }(d)$ and $c_{i}^{\max }(d)$ such that the bound state exists only if $c \in\left(c_{i}^{\min }, c_{i}^{\max }\right)$. The energy of the bound state "sinks" into the continuous spectrum at these points:

$$
\begin{aligned}
& \lim _{c \rightarrow c_{i}^{\min }} E_{i}=1, \\
& \lim _{c \rightarrow c_{i}^{\max }} E_{i}=-1 .
\end{aligned}
$$

4. By analogy for fixed $c$ there are $d_{i}^{\min }(c)$ and $d_{i}^{\max }(c)$ and the bound state exists only if $d \in\left(d_{i}^{\min }, d_{i}^{\max }\right)$.

5. $d_{i}^{\min }(c)=\frac{2 i \pi}{\sqrt{c(c+2)}}$ and $d_{i}^{\max }(c)=\frac{(2 i+1) \pi}{\sqrt{c(c-2)}}$. When $c<2$ the value $d_{i}^{\max }(c)=\infty$. The energies are numbered from 0 to $\infty$.

We can formally consider the points with parameters $c, d_{i}^{\min }(c)$ or $c, d_{i}^{\max }(c)$ as exceptional points, but in these points no complexification takes place. The spin-1/2 particle in a square well thus behaves "correctly" and exhibits no complexification for strong potential; this can be placed in contrast to a scalar particle in an analogous potential described in the next subsection.

\subsection{Klein-Gordon square well}

It is well known that a scalar particle is described by the Klein-Gordon equation. As for the involved potential we will consider the minimal coupling, and thus the equation for stationary states has the form 

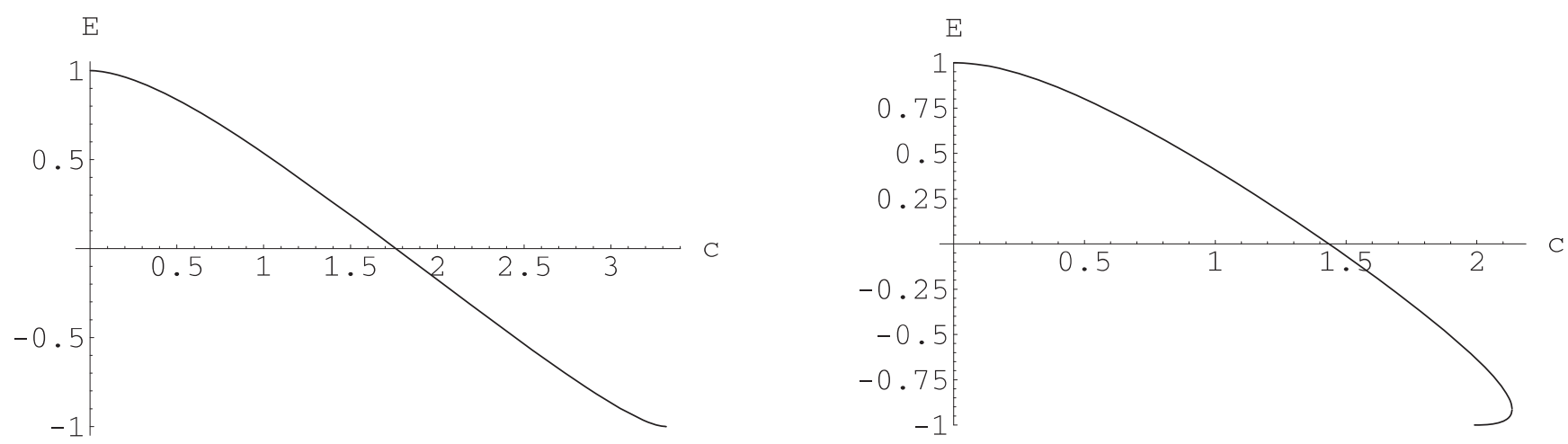

Fig. 3: The lowest energy level plotted against depth $c$ with fixed width $d=1.5$ for relativistic square wells. The spin-1/2 case on the left hand side does not complexify while the spinless case on the right hand side does; in the latter graph the two levels that merge in the EP are shown.

$$
\left(\left(E+c \chi_{(0, d)}(x)\right)^{2}-\partial_{x}^{2}-M^{2}\right) \psi(x)=0,
$$

where $c$ is the well's depth and $d$ its width.

It is worth mentioning that it is possible to describe system (23) by a Hamiltonian of form (1) (see [13]). The price paid is that one must introduce a two-component formalism, which leads to some ambiguities as long as the step from one to two components is not unique, and the resulting Hamiltonian is non-Hermitian $^{(10)}$. Since we are mainly interested in the spectrum we will not follow this way.

After some manipulations with matching conditions at 0 and $d$ and logarithming as in the Dirac case, it is easy to obtain the secular equation for the eigenvalues, which reads

$\arctan \sqrt{\frac{(1+E)(1-E)}{(c+E-1)(c+E+1)}}=\frac{d}{2} \sqrt{(c+E)^{2}-1} \bmod \frac{\pi}{2}$.

Although (24) resembles (21), the solutions to it behave differently at larger values of $c$ and $d$. In addition to levels analogous to those of the Dirac Hamiltonian, there is a second set of energies emerging from the lower continuum with increasing $c$ or $d$ that merge with the former in a traditionally shaped exceptional point (Fig. 3). This effect is enabled by the non-self-adjointness of the Hamiltonian, in contrast with the Dirac problem.

\subsection{Relativistic coulomb Hamiltonian}

The last example is intended to show that also the Dirac equation can lead to complex energies. It is the well-known radial Coulomb Hamiltonian

$$
\mathrm{H}=\left(\begin{array}{cc}
-\frac{c}{r}-m & -\partial_{r}-\frac{\kappa}{r} \\
\partial_{r}-\frac{\kappa}{r} & -\frac{c}{r}+m
\end{array}\right),
$$

where $\kappa \in\{1,2,3, \ldots\}$ characterises the angular momentum and $m$ is the mass, once more without loss of generality set to one in what follows. We will not discuss the algorithm of the solution since the reader is assumed to know it, and instead we write down the resulting formula

$$
\begin{aligned}
& E_{n}=\left(1+\frac{c}{n-\kappa+\gamma}\right)^{-\frac{1}{2}}, \\
& \gamma=\sqrt{\kappa^{2}-c^{2}} .
\end{aligned}
$$

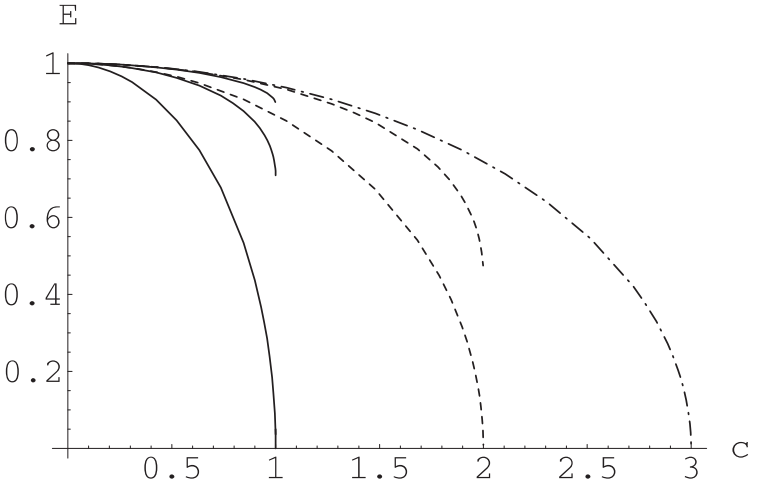

Fig. 4: Six lowest real energies of the Dirac Hamiltonian with Coulomb potential. The line styles distinguish between different values of $\kappa$.

Obviously $c>\kappa$ leads to complex values of $E_{n}$ and all the levels with the same $\kappa$ complexify simultaneously. The energy dependence is clearly of square-root type, however only one real eigenvalue exists at $c<c_{\mathrm{ep}}$ for each complex pair at $c>c_{\text {ep }}$. To see how this is possible for the symmetric Hamiltonian (25) one needs to examine it more closely. We can restrict ourselves to $s$-states $(\kappa=1)$ because these complexify first and there is no qualitative difference for higher $\kappa$.

The Hamiltonian's domain of definition trivially must be a subset of

$D=\left\{\psi \in L_{2}\left(\mathbb{R}^{+}\right) \oplus L_{2}\left(\mathbb{R}^{+}\right) \mid \mathrm{H} \psi \in L_{2}\left(\mathbb{R}^{+}\right) \oplus L_{2}\left(\mathbb{R}^{+}\right)\right\}$,

(the existence of $\mathrm{H} \psi$ for all $\psi \in D$ is understood). If one has to establish the self-adjointness of $\mathrm{H}$ one has first to apply per partes integration on the integrals of wave function products and demand vanishing of boundary terms. The boundary at $r=\infty$ causes no complications because $\psi(r) \rightarrow 0$ for $r \rightarrow \infty$ trivially due to the square integrability. One must be more careful at $r \rightarrow 0$. The square integrability of $\psi$ itself clearly does not forbid non-zero values at origin, however square integrability of $\mathrm{H} \psi$ can do better. To see whether there are functions from $D$ with non-zero limit at $r=0$ one may check the asymptotics of $\mathrm{H} \psi$; around zero one can disregard the mass-proportional terms, and one has

$$
\mathrm{H}\left(\begin{array}{c}
\psi_{1} \\
\psi_{2}
\end{array}\right)=\left(\begin{array}{c}
-c r^{-1} \psi_{1}-r^{-1} \psi_{2}-\psi_{2}^{\prime} \\
\psi_{1}^{\prime}-r^{-1} \psi_{1}-c r^{-1} \psi_{2}
\end{array}\right) .
$$


This two-component function can be square-integrable

1. either if $\psi \propto r^{\alpha}$ with $\alpha>1 / 2$ (and thus $\psi(0)=0$ ), because then both $\psi^{\prime}$ and $\psi / r$ are proportional to $r^{\alpha+1}$ and therefore square integrable

2. or if the whole expression (29) vanishes.

The second case leads to the differential equation for asymptotical behaviour of $\psi$ near the origin, and the solution to it is

$$
\psi \propto r^{ \pm \gamma}
$$

with the same $\gamma>0$ as in (27) $-\kappa$ still being equal to 1 . The solution proportional to $r^{+\gamma}$ is not interesting for being zero at $r=0$ but the solution $r^{-\gamma}$ diverges. For $\gamma>1 / 2$ it is not square integrable and can thus be ruled out, but if $\gamma<1 / 2$ (it is $c>\sqrt{3} / 2$ ) this solution lies in $D$. In other words, in this case there are functions from $D$ which do not vanish at $r=0$ and if we make $D$ the definition domain of $\mathrm{H}$ the operator would not be symmetric because of the non-zero boundary term in per partes integration.

After restricting the domain of $\mathrm{H}$ to functions which vanish in the origin the operator is symmetric with point spectrum (26), but still not self-adjoint, since the domain of the adjoint operator would be the entire set $D$, which is now for $c>\sqrt{3} / 2$ different from Dom $\mathrm{H}$.

From the previous discussion it follows that there are two main differences between square-well and Coulomb interaction within the framework of the Dirac equation. The first, connected with the long range of Coulomb potential, is that the eigenvalues do not emerge from the upper continuum as the strength of the interaction increases (as they do in the case of a square well) but they all exist for any non-zero $c$. A similar difference is also present in a non-relativistic treatment of the problems. The second difference, more interesting for the purposes of this paper, the existence of complexification at a certain value of $c$, is a consequence of the $1 / r$ singularity at the origin. This complexification is of an extraordinary type within the family of $\mathcal{P} \mathcal{T}$-symmetric models with only one real eigenvalue forming a complex pair ${ }^{(11)}$.

\section{Summary}

The purpose of this paper was two-fold: First to discuss the simple principles behind the complexification of eigenvalues and to show the connection between avoided level crossings in the Hermitian framework and avoided diagonalisable EPs in $\mathcal{P}$-symmetry. And second, to show that in less standard situations (where standard means a matrix or a Schrödinger Hamiltonian), there are more ways in which the eigenvalues can cross the boundary between the physical world and the complex realm. It is by no means intended to state that the few examples presented here constitute in any sense a complete list of what can happen. Rather the aim of the article was to illustrate that some quite ordinary systems can behave contrary to the usual "PT-symmetric intuition".

\section{Remarks}

${ }^{(1)}$ It may be useful to note that a systematic approach to $\mathcal{P}$-symmetry cannot be probably achieved without use of the Krein space concept [1]. When the spectrum of a Krein space operator is real, a transformation to a Hilbert space operator exists and the $\mathcal{P} \mathcal{T}$-symmetric system can be treated as in standard quantum mechanics.

(2) Some authors distinguish between "exceptional" and "diabolic" points regarding the type of complexification. Here we use the first term for all types.

${ }^{(3)}$ Usually but not necessarily the standard realisations of $\mathcal{P}$ and $\mathcal{T}$ are used. A particularly useful non-standard choice is $\mathcal{P}=\mathrm{I}$, which identifies symmetric and $\mathcal{P} \mathcal{T}$-symmetric operators.

(4) This need not to be valid for general $\mathrm{H}_{0}$ and $\mathrm{V}$, but most physical systems do not spoil the assumption. In many situations it can be proved through perturbation theory.

${ }^{(5)}$ The mapping $S$ is a bijection which creates $N^{2}$-dimensional vector from $N^{2}$ independent real parameters determining the Hermitian $N \times N$ matrix, i.e. the real diagonal matrix elements and real and imaginary parts of the entries above the diagonal.

${ }^{(6)}$ Such matrices obviously exist. A particular example can be easily constructed in the diagonal basis of $\mathrm{H}_{0}$ : Any matrix which is diagonal in this basis and has values $v_{i i}=\left(E_{\mathrm{ep}}-\left(\mathrm{H}_{0}\right)_{i i}\right) c_{\mathrm{ep}}^{-1}$ at least for two different $i \in\{1 \ldots N\}$ is good enough.

${ }^{(7)}$ We suppose the measure in the space of matrices is generated by an Euclidean norm $\|\mathrm{A}\|=\sum A_{i j}^{2}$ or equivalent. On contrary there can be reasons for choosing measures concentrated on less-dimensional subspaces - usually because of some symmetry prescription for the „randomly choosen“ Hamiltonian. Under such conditions the level crossings are not forbidden.

${ }^{(8)}$ It is the most general $\mathcal{P} \mathcal{T}$-symmetric two-dimensional matrix with respect to $\mathcal{P}$ defined by Pauli matrix $\sigma_{3}$ and $\mathcal{T}$ being simply complex conjugation.

${ }^{(9)}$ Strictly speaking the system defined by (15) is not of type (1). But the linear parametrisation is still valid in a generalised sense, for the Hamiltonian's associated sequilinear form.

${ }^{(10)}$ And also not manifestly Lorentz covariant.

${ }^{(11)}$ To be more specific, the statement is true if the boundary condition $\psi(0)=0$ is given. Without it, demanding only square integrability of $\psi$ and $\mathrm{H} \psi$, one gets a clearly non-physical situation: if $c>\sqrt{3} / 2$, then all real numbers become eigenvalues with eigenfunctions proportional to confluent hypergeometric function $U$ multiplied by decaying exponential.

\section{References}

[1] Mostafazadeh, A.: Krein-Space Formulation of PT-Symmetry, $\mathcal{C}$ P -Inner Products, and Pseudo-Hermiticity, Czech. J. Phys. Vol. 56 (2006), p. 919. 
[2] Heiss, W. D., Müller, M., Rotter, I.: Collectivity, Phase Transitions and Exceptional Points in Open Quantum Systems, Phys. Rev. Vol. E58 (1998), p. 2894.

[3] Cejnar, P., Heinze, S., Dobeš, J.: Thermodynamic Analogy for Quantum Phase Transitions at Zero Temperature, Phys.Rev. Vol. C71 (2005), p. 011304.

[4] Günther, U., Stefani, F., Gerbeth, G.: The MHD $\alpha^{2}-$ Dynamo, $\mathbb{Z}^{2}$-Graded Pseudo-Hermiticity, Level Crossings and Exceptional Points of Branching Type. Czech. J. Phys. Vol. 54 (2004), p. 1075.

[5] Günther, U., Stefani, F., Znojil M.: MHD $\alpha^{2}$-Dynamo, Squire Equation and $\mathcal{P}$ - -Symmetric Interpolation between Square Well and Harmonic Oscillator, J. Math. Phys. Vol. 46 (2005), p. 063504.

[6] Günther, U., Rotter, I., Samsonov, B. F.: Projective Hilbert Space Structures at Exceptional Points, arXiv:0704.1291.

[7] Mostafazadeh, A., Batal, A.: Physical Aspects of Pseudo-Hermitian and $\mathcal{P} \mathcal{T}$-Symmetric Quantum Mechanics, $J$. Phys. Vol. A37 (2004), p. 11645.

[8] Quesne, C., Bagchi, B., Mallik, S., Bíla, H., Jakubský, V., Znojil, M.: PT-Supersymmetric Partner of a Short-Range Square Well, Czech. J. Phys. Vol. 55 (2005), p. 1161.
[9] Mostafazadeh, A.: Exact $\mathcal{P}$ T-Symmetry is Equivalent to Hermiticity, J.Phys. Vol. A36 (2003), p. 7081.

[10] Bender, C. M., Boettcher, S., Meisinger, P.: PT-symmetric Quantum Mechanics, J. Math. Phys. Vol. 40 (1999), p. 2201.

[11] Krejčiřík, D., Bíla, H., Znojil, M.: Closed Formula for the Metric in the Hilbert Space of a $\mathcal{P}$-Symmetric Model, J. Phys. Vol. A39 (2009). p. 10143.

[12] Lévai, G.: Comparative Analysis of Real and PT-Symmetric Scarf Potentials, Czech. J. Phys. Vol. 56 (2006) p. 953 .

[13] Feshbach, H., Villars, F.: Elementary Relativistic Wave Mechanics of Spin 0 and Spin 1/2 Particles, Rev. Mod. Phys. Vol. 30 (1958).

Mgr. Hynek Bíla

phone: +420266 173280

email: hynek.bila@ujf.cas.cz

Nuclear Physics Institute

Academy of Science of the Czech Republic

25068 Rež, Czech Republic 